\title{
Remote ischemic preconditioning in myocardial protection in hemodialysis patients
}

This article was published in the following Dove Press journal: International Journal of General Medicine

\author{
Marcelo R Bacci' \\ Livia Y Vasconcelos' \\ Neif Murad ${ }^{2}$ \\ Antonio Carlos P Chagas ${ }^{2}$ \\ Ana Carolina Capuano' \\ Beatriz CA Alves ${ }^{3}$ \\ Edimar C Pereira ${ }^{4}$ \\ Ligia A Azzalis ${ }^{4}$ \\ Virginia $B C$ Junqueira ${ }^{4}$ \\ Fernando LA Fonseca ${ }^{3,4}$ \\ 'Department of General Practice, \\ ${ }^{2}$ Department of Cardiology, ${ }^{3}$ Clinical \\ Analysis Laboratory of Faculdade de \\ Medicina do ABC, Santo André, SP, \\ Brazil; ${ }^{4}$ Department of Pharmaceutical \\ Sciences, Universidade Federal de Sao \\ Paulo, Diadema, SP, Brazil
}

Correspondence: Marcelo R Bacci Department of General Practice, Faculdade de Medicina do ABC, Av. Principe de Gales n 82I, Vila Valparaíso, Santo André, SP, ZIP 09060-650, Brazil

$\mathrm{Tel}+55$ II 981937005

Emailmrbacci@yahoo.com
Background: Remote ischemic preconditioning (RIPC) is a procedure that generates a brief period of ischemia followed by reperfusion. The role of RIPC in protecting myocardial ischemia during hemodialysis is not yet established. The aim of the study was to evaluate RIPC myocardial protection as evaluated by ultrasensitive I troponin in hemodialysis outpatients.

Patients and methods: A double-blind randomized trial with two groups: intervention submitted to RIPC and control group without RIPC. Intervention group received RIPC in three consecutive hemodialysis sessions. Blood samples were taken before and after each session. Blood urea nitrogen for calculation of single-pool Kt/v and ultrasensitive I troponin were measured to evaluate dialysis adequacy and myocardial injury.

Results: A total of 47 patients were randomized. About $60.8 \%$ were men and $54 \%$ were diabetic. The mean single-pool Kt/v was 1.51 in the intervention group and 1.49 in control. The ultrasensitive troponin I measured no significant change from the time of collection: before or after dialysis.

Conclusion: The RIPC applied in three consecutive sessions did not demonstrate superiority to control, therefore another study tested RIPC in 12 consecutive sessions with a positive result in myocardial protection. In our study, more than half of the patients were diabetic. Diabetic patients have a trend to show a lower response to RIPC because of the greater presence of collateral coronary circulation. In summary, in this model there was no interference of RIPC in ultrasensitive troponin I values, but troponin had a high negative predictive value for myocardial infarction in all tested models.

Keywords: hemodialysis, chronic kidney disease, cardiac troponin, remote ischemic preconditioning, myocardial infarction

\section{Introduction}

Remote ischemic preconditioning (RIPC) is defined as brief transient episodes of ischemia followed by a prolonged sustained episode of ischemia and reperfusion in the heart. It was first described by Murry et al in 1986, when they showed that brief ischemic episodes in dogs by the experimental occlusion of the left coronary artery reduced infarct extension caused by the occlusion of the circumflex artery and subsequent reperfusion. ${ }^{1}$ It was observed that these induced ischemic periods followed by reperfusion promote cardioprotection in situations in which this prolonged ischemic event is expected, like in cardiac surgeries, transplants or even percutaneous coronary angioplasty. $^{2}$

Kadkhodaee et al reported protective effects in the kidney by pre- and postconditioning in ischemic situations and induced reperfusion. ${ }^{3}$ Besides all other risk 
factors, patients on hemodialysis have a common complication from the procedure associated with myocardial ischemia. With the prolonged decrease of oxygen supply, a left ventricular dysfunction can be observed in the long run, resulting in cardiac insufficiency and an increase in mortality. ${ }^{4}$

A recent study reveals that conventional hemodialysis, besides being associated with endothelial injury, oxidative stress and high inflammatory activity, can induce microcirculation injury with consequent critical ischemia and tissue damages during the procedure., ${ }^{4} 5$

It is known that cardiac troponin I (cTnI) and cardiac troponin T (cTnT) levels are usually elevated and related to mortality in such cases. A recent meta-analysis concluded that the elevation of cTnI and cTnT represents a 2.64- and 1.74-fold increase in mortality, respectively. ${ }^{6}$

The aim of this study was to analyze the RIPC effect in myocardial injury by means of the measurement of cTnI in patients with chronic kidney disease on hemodialysis.

\section{Patients and methods Study design}

This is a randomized clinical trial. Patients were divided into two groups: experiment and control. Randomization was performed according to the list generated by the website www.randomizatiom.com, which was accessed only by the nurse in charge of the dialysis. Researchers remained blinded as to which group each patient belonged to. Patients on medications for the treatment of pre-existing diseases were not washed out.

Hemodialysis sessions were set to 3:30 hours, with blood flow of $300 \mathrm{~mL} / \mathrm{min}$, dialysate flow of $500 \mathrm{~mL} / \mathrm{min}$, sodium concentration of $140 \mathrm{mEq} / \mathrm{L}$ and bicarbonate of $34 \mathrm{mEq} / \mathrm{L}$. The ultrafiltration value was defined according to the individual need of each patient. Written informed consent was obtained from each participant included in this study; this study and its protocol were conducted in accordance with the Declaration of Helsinki, and the study was approved by the Faculdade de Medicina do ABC Ethics Committee (CAAE number 45920015.5.0000.0082).

\section{Study interventions}

The ischemic preconditioning consisted of a 5-minute interruption of the brachial blood flow using a Tycos sphygmomanometer (Welch Allyn, Skaneateles Falls, NY, USA) inflated to $200 \mathrm{mmHg}$ or until the distal blood flow was interrupted; subsequent deflation followed with the same rest period, and the procedure was repeated twice, totaling 30 minutes of intervention. The contralateral arm was chosen, next to the functioning arteriovenous fistula, and in the absence of the fistula, the right arm was used. The intervention occurred in the beginning of the dialysis session. The intervention group underwent three consecutive sessions of RIPC, whereas control group underwent none.

\section{Interesting outcomes}

The primary outcome was the decrease in ultrasensitive cTnI level when intervention and control groups were compared. The secondary outcome was mortality by any cause at time points 30 and 90 days, hospitalization due to cardiovascular event defined as acute coronary syndrome, pulmonary thromboembolism or cerebrovascular accident.

\section{Variables of interest}

The analysis of biochemical markers was carried out using blood samples collected at 2 and 7 days prior to the allocation, in the beginning of the first hemodialysis session, after the initial allocation.

Plasma cTnI levels were measured by ELISA assay, and their cutoff values were defined according to the constructed calibration curve. Values above the 99th percentile were considered altered.

For dialysis adequacy, the values of single-pool Kt/V and the urea reduction ratio (URR) were considered adequate if they were $\geq 1.2$ and $65 \%$, respectively.

The analysis of the inflammatory markers was performed using the ELISA method. Cutoff values were set on a methodby-method basis according to each used kit, following each manufacturer's instructions.

\section{Statistical analysis}

This was a 40-patient pilot study. The sample was divided into two groups: 20 in the control group and 20 in the intervention group.

Qualitative variables were presented with absolute and relative frequencies and analyzed by the chi-square test. Shapiro-Wilk test was used to verify the normality of quantitative variables. As the distribution was not normal, median values, along with the respective $95 \%$ confidence intervals, were used. Quantitative variable between groups were analyzed with the Mann-Whitney test, and Wilcoxon test was applied to verify if there were differences in the collections before and after each moment, confidence interval was $95 \%$. Data Analysis and Statistical Software for Professionals (Stata), version 11.0, was the statistical package used. 


\section{Results}

The mean age in the control group was 63.9 years (SD \pm 15.8 ), and in the intervention group it was 52.1 years $(\mathrm{SD} \pm 21.5)$. All of the patients from both groups suffered from systemic hypertension. Diabetes was present in $60.8 \%$ of the patients in the intervention group and in $65.2 \%$ in the control group.

Table 1 shows the characterization of the samples, taking parameters like gender, Kt/V, hemoglobin and ferritin into consideration.

Table 2 shows the mean values of troponin pre- and post-dialysis in control and intervention groups. Interestingly, between the moments of pre- and post-dialysis the significance value was $<0.05(p<0.05)$ according to the Wilcoxon test, demonstrating that the RIPC did not have the expected effect.

In Table 3 it can be observed that there were no significant variations in the point estimation for urea, URR and troponin between control and intervention groups.

Table I Characterization of samples according to the studied groups

\begin{tabular}{|c|c|c|c|}
\hline \multirow{2}{*}{$\begin{array}{l}\text { Studied } \\
\text { variables }\end{array}$} & \multicolumn{2}{|l|}{ Groups } & \multirow[t]{2}{*}{$p^{*}$} \\
\hline & Control & Intervention & \\
\hline \multicolumn{4}{|l|}{ Gender, n (\%) } \\
\hline Female & $9.0(40.9)$ & $8.0(34.8)$ & $0.672 *$ \\
\hline Male & I $3.0(59.1)$ & $15.0(65.2)$ & \\
\hline $\mathrm{Kt} / \mathrm{V}$ & $\mathrm{I} .5(\mathrm{I} . \mathrm{I}-\mathrm{I} .5)$ & $1.5(1.1-1.6)$ & $0.846 * *$ \\
\hline Hemoglobin, g/d & $-10.6(10.4-11.5)$ & II.I (10.8-|| I.6) & $0.190 * *$ \\
\hline Albumin, g/dL & $3.8(3.6-4.0)$ & $4.0(3.9-4.1)$ & $0.010^{* * *}$ \\
\hline Ferritin, ug/L & $560.0(364.9-563.5)$ & $375.0(278.1-391.7)$ & $0.076 * *$ \\
\hline
\end{tabular}

Notes: Confidence interval of $95 \%$ shown in brackets; *chi-square; ** MannWhitney test.

\section{Discussion}

It is known that chronic kidney disease patients on hemodialysis, even those without acute coronary disease, have high serum levels of troponin.

So far, very few management strategies have been proposed to reduce myocardial damage caused by hemodialysis. Remote ischemic preconditioning is one of the propositions. ${ }^{7,8}$

Troponin is the biomarker of choice for the analysis of myocardial ischemia due to the fact that it is an enzyme specific to the cardiac muscle that plays an important role in the muscle contraction process; moreover, it practically has no relation with skeletal muscles. Most of the studies use troponin $\mathrm{T}(\mathrm{TnT})$ as a marker; studies using troponin I (TnI) are not only few but also short-term, with a small number of patients included. ${ }^{9}$

A 2012 study on the predictive value of troponin in patients on hemodialysis revealed a strong discrepancy between $\mathrm{TnT}$ and $\mathrm{TnI}$ values, which can be explained by their molecular mechanisms, like structure, molecular weight and half-life. ${ }^{10}$ Besides, the kinetics of enzyme release in the circulation after the alterations in myocyte membrane permeability can be different, and there is a divergence between the clearance of both enzymes Once released in the circulation, TnI becomes more susceptible to modifications owing to phosphorylation, oxidation and proteolysis.

Factors like aggressive ultrafiltration and intradialytic hypotension are associated with the aggravation of the myocardial ischemic condition during a hemodialysis session owing to the significant alterations in the coronary flow. Repeated injuries induced by ischemia followed by

Table 2 Point estimation (interval estimation) of troponin concentration according to the moments in control and intervention groups

\begin{tabular}{|c|c|c|c|c|c|c|}
\hline \multirow[t]{2}{*}{ cTn } & \multicolumn{3}{|l|}{ Control } & \multicolumn{3}{|l|}{ Intervention } \\
\hline & Pre & Post & $\mathbf{p}^{*}$ & Pre & Post & $p^{*}$ \\
\hline Troponin I & $0.010(0.006-0.016)$ & $0.009(0.006-0.023)$ & 0.631 & $0.007(0.006-0.012)$ & $0.007(0.006-0.01 \mathrm{I})$ & 0.152 \\
\hline Troponin 2 & $0.010(0.006-0.014)$ & $0.011(0.006-0.018)$ & 0.634 & $0.009(0.006-0.017)$ & $0.006(0.006-0.014)$ & 0.987 \\
\hline Troponin 3 & $0.011(0.006-0.018)$ & $0.013(0.007-0.027)$ & 0.024 & $0.007(0.006-0.018)$ & $0.006(0.006-0.009)$ & 0.136 \\
\hline
\end{tabular}

Notes: Values expressed in median (confidence interval of 95\%); *Wilcoxon test.

Table 3 Point estimation of concentration variation of urea, troponin and urea reduction ratio between groups

\begin{tabular}{lll}
\hline Markers & Groups & \multicolumn{1}{c}{$\boldsymbol{P}^{* *}$} \\
\cline { 2 - 3 } & Control & Intervention \\
\hline Urea* & $63.50(36.86-89.35)$ & $75.75(35.86-104.52)$ \\
Troponin* & $-0.001(-0.0064-0.0021)$ & $0.001(-0.0014-0.0014)$ \\
Urea reduction ratio * & $-0.650(-8.074-11.427)$ & $4.500(-8.090-13.901)$
\end{tabular}

Notes: Values expressed in median (confidence interval of 95\%); *variation between the first and last collection; **Wilcoxon test. 
reperfusion cause alterations in motility and loss of contractility, which may lead to myocardial fibrosis and irreversible left ventricular systolic dysfunction. ${ }^{11}$

Burton et al showed that nearly $50 \%$ of patients on hemodialysis developed alterations in regional myocardial motility, and also that the mortality rate among these patients increased in the subsequent 12 months. ${ }^{4}$

No RIPC benefits, performed in three consecutive hemodialysis sessions in this study, could be observed. However, Park et al reported its beneficial effects in 12 consecutive hemodialysis sessions, when a decrease in TnT levels could be observed. ${ }^{12}$

This study faced some limitations. First, the number of sessions was lower than in other published studies, and it is not surely known how many interventions are necessary to obtain such benefits. The presence of diabetes is a positive predictive factor for higher cardiovascular mortality. Moreover, coronary collateral circulation in diabetic patients is higher due to the presence of ischemia, a factor that may be responsible for the lower response rate in patients undergoing RIPC. The studied sample included a diabetic population of $>50 \%$. ${ }^{5,13}$

In sum, in the model of three consecutive hemodialysis sessions, no significant reduction in ultrasensitive troponin I concentrations could be observed when remote ischemic preconditioning was applied.

\section{Disclosure}

The authors report no conflicts of interest in this work.

\section{References}

1. Murry CE, Jennings RB and Reimer KA. Preconditioning with ischemia: a delay of lethal cell injury in ischemic myocardium. Circulation 1986;74(5):1124-1136.

2. Przyklenk K, Bauer B, Ovize M, Kloner RA, Whittaker P. Regional ischemic preconditioning protects remote virgin myocardium from subsequent sustained coronary occlusion. Circulation 1993;87(3):893-899.

3. Kadkhodaee M, Seifi B, Najafi A, Sedaghat Z. First report of the protective effects of remote per- and postconditioning on ischemia/ reperfusion-induced renal injury. Transplantation. 2011;92(10):e55.

4. Burton JO, Jefferies HJ, Selby NM, McIntyre CW. Hemodialysisinduced cardiac injury: determinants and associated outcomes. Clin J Am Soc Nephrol. 2009;4(5):914-920.

5. Benhamou Y, Begarin L, David N et al. Detection of microcirculatory impairment by transcutaneous oxymetry monitoring during hemodialysis: an observational study. BMC Nephrol. 2014;15(4):1-8.

6. Kalaji FR, Albitar S. Predictive value of cardiac Troponin T and I in hemodialysis patients. Saudi J Kidney Dis Transpl. 2012; 23(5):39-945.

7. Gassanov N, Nia AM, Caglayan E, Er F. Remote ischemic preconditioning and renoprotection: from myth to a novel therapeutic option? J Am Soc Nephrol. 2014;25(2):216-224.

8. Heusch G. Cardioprotection: chances and challenges of its translation to the clinic. Lancet. 2013; 381(9861):166-175.

9. Alam A, Palumbo A, Mucsi I, Barre PE, Sniderman AD. Elevated troponin I levels but not low grade chronic inflammation is associated with cardiac-specific mortality in stable hemodialysis patients. $B M C$ Nephrol. 2013;14:247-253.

10. Artunc F, Mueller C, Breidthardt T, et al. Sensitive troponins - which suits better for hemodialysis patients? Associated factors and prediction of mortality. PLoS One. 2012;7(10):1-9.

11. Przyklenk K, Bauer B, Ovize M, Kloner RA, Whittaker P. Regional ischemic preconditioning protects remote virgin myocardium from subsequent sustained coronary occlusion. Circulation. 1993;87(3):893-899.

12. Park J, Ann SH, Chung HC et al. Remote ischemic preconditioning in hemodialysis: a pilot study. Heart Vessels. 2014;29(1):58-64.

13. Venugopal V, Laing CM, Ludman A, Yellon DM, Hausenloy D. Effect of remote ischemic preconditioning on acute kidney injury in nondiabetic patients undergoing coronary artery bypass graft surgery: a secondary analysis of 2 small randomized trials. Am J Kidney Dis. 2010;56(6):1043-1049.
International Journal of General Medicine

\section{Publish your work in this journal}

The International Journal of General Medicine is an international, peer-reviewed open-access journal that focuses on general and internal medicine, pathogenesis, epidemiology, diagnosis, monitoring and treatment protocols. The journal is characterized by the rapid reporting of reviews, original research and clinical studies across all disease areas.

\section{Dovepress}

The manuscript management system is completely online and includes a very quick and fair peer-review system, which is all easy to use. Visit http://www.dovepress.com/testimonials.php to read real quotes from published authors. 